\title{
Crenças, Atitudes e Práticas dos Profissionais de Recursos Humanos Diante do Cumprimento da Lei de Cotas*
}

\author{
Michelli Godoi Rezende* \\ Maria Nivalda de Carvalho-Freitas* \\ Marcos Vieira-Silva* \\ Universidade Federal de São João del-Rei, São João Del Rei, MG, Brasil
}

\begin{abstract}
RESUMO
Esta pesquisa buscou verificar se existe relação entre crenças, atitudes e práticas dos profissionais de Recursos Humanos em relação à inclusão de pessoas com deficiência (PCD) nas organizações. Participaram do estudo 55 profissionais de Recursos Humanos filiados à Associação Brasileira de Recursos Humanos de Minas Gerais. Na pesquisa foram utilizados métodos quantitativos de coleta e análise dos dados. De forma geral, constatou-se que a utilização dos construtos: concepções de deficiência, ações consideradas possíveis para garantir a inclusão de PCD no trabalho e percepção das práticas de RH utilizadas nas organizações contribuíram para a identificação de atitudes diferenciadas dos profissionais de RH frente à inclusão.
\end{abstract}

Palavras-chave: inclusão; pessoas com deficiência; trabalho; práticas de RH.

\begin{abstract}
Attitude of Human Resource Professionals on the fulfillment of the Law of Quotas

This study aimed to verify if beliefs, attitudes and Human Resources practices are correlated, considering the inclusion of people with disability (PWD) in organizations. The study was carried out with $55 \mathrm{HR}$ professionals affiliated to Brazilian Association of Human Resource of Minas Gerais, Brazil. Quantitative methods were used to collect and analyze data. In general, it was found that the use of the constructs: conceptions of disability, possible actions to ensure the inclusion of PWD at work and perception of HR practices contributed to the identification of different attitudes of $\mathrm{HR}$ professionals related to inclusion.
\end{abstract}

Keywords: inclusion; people with disabilities; work; HR practices.

A necessidade de se garantirem direitos iguais de acesso e oportunidades a todas as pessoas é prioridade presente em políticas públicas de vários países do mundo (Goss, D., Goss, F., \& Adam-Smith, 2000; Blanchard, 2001; Oliveira, Goulart Jr., \& Fernandes, 2009).

Nessa perspectiva, a Lei de Cotas (Lei 8.213/91) no Brasil caracteriza-se por ser uma ferramenta avançada de proteção e apoio à pessoa com deficiência $(\mathrm{PCD})^{1}$, mas ainda apresenta sérias lacunas, preconceitos e barreiras que impedem sua efetiva aplicabilidade (Ribeiro \& Carneiro, 2009; Garcia, 2014). A Lei de Cotas, ou Reserva Legal de Cargos, estabelece que empresas com 100 ou mais funcionários sejam obrigadas a contratar, em seu quadro efetivo, pessoas com deficiência. No entanto, a simples inserção de PCD nas empresas não significa garantia de um processo verdadeiramente inclusivo no trabalho.

Para Sassaki (2006), "a inserção não exige nenhuma modificação por parte da sociedade em termos de modificação de atitudes, de espaços físicos, de objetos e práticas sociais" (p. 35). Por outro lado, a inclusão parte do pressuposto de que a sociedade tem importante papel no sentido de modificar e oferecer os me-

\footnotetext{
Endereço para correspondência: Michelli Godoi Rezende - michelligodoi@gmail.com

Endereço para correspondência: Maria Nivalda de Carvalho-Freitas - nivalda@ufsj.edu.br

* Endereço para correspondência: mvsilva@ufsj.edu.br - Marcos Vieira-Silva
} 
canismos necessários para que a pessoa com deficiência possa se "desenvolver e exercer a cidadania" (Sassaki, 2006, p. 43).

Dados recentes divulgados pelo Instituto Brasileiro de Geografia e Estatística (IBGE, 2010) revelam que, de uma população de quase 191 milhões de pessoas, mais de 45 milhões possuem algum tipo de deficiência, o que representa $23,91 \%$ da população brasileira. Conforme estudo realizado pelo Instituto Ethos (2010), apesar de constatado um aumento no número de empresas que têm em seu quadro efetivo pessoas com deficiência, esse número ainda é pouco representativo perante o que prevê a legislação. No Brasil, de acordo com os dados da Relação Anual de Informações Sociais (RAIS) [Ministério do Trabalho e Emprego (MTE), 2011], somente 0,7\% do total de vínculos empregatícios formais no ano de 2011 foram de pessoas com deficiência, o que denota baixos índices de empregabilidade para essa parcela da população. Tal índice mantém-se estável desde 2010.

A deficiência tem sido historicamente objeto de discriminação e preconceito (Aranha, 1995; Pessotti, 1984; Carvalho-Freitas \& Marques, 2007). E a partir desse entendimento preconceituoso sobre as PCD, barreiras foram construídas e consolidadas, podendo ser encontradas até hoje nos mais diversos ambientes sociais e sob variadas formas, sendo a barreira atitudinal o principal obstáculo para a inclusão (Lima, Guedes, L. C., \& Guedes, M. C., 2010; Suzano, 2011).

A atitude tem uma dimensão avaliativa que varia em direção e intensidade. Tal direção pode ser antagônica, como positiva-negativa, concordância-discordância e aceitação-rejeição. Já a intensidade envolve graus diferentes de aceitação, rejeição ou discordância em relação ao objeto (Tanaka, 2007). As barreiras atitudinais, por sua vez, estão relacionadas a uma predisposição negativa às pessoas com deficiência na medida em que estas se distanciam do padrão de normalidade comumente aceito pela sociedade.

Para Finkelstein (1980), a deficiência nada mais é do que uma construção social, pois é a sociedade que estabelece os padrões de normalidade, criando o paradoxo entre pessoas com e sem deficiência, e, a partir disso, julga, segundo seus critérios, aqueles que não se encaixam nesses padrões. $\mathrm{O}$ autor enfatiza a necessidade de desviar o foco para os aspectos sociais, abrindo novos caminhos para eliminar o preconceito e promover a acessibilidade e a efetiva inclusão de pessoas com deficiência.
De acordo com pesquisa realizada por CarvalhoFreitas e Marques (2010), as possibilidades para a inserção de PCD em dado período histórico são decorrentes da forma predominante de ver a deficiência. Frente a essa perspectiva, diferentes crenças ou concepções de deficiência caracterizam as ações historicamente voltadas para as pessoas com deficiência (Carvalho-Freitas, 2007) e que ainda hoje podem ser observadas nos amplos contextos sociais. São elas:

$\checkmark$ Concepção espiritual da deficiência: corresponde à percepção sobrenatural ou mítica da deficiência; as PCD vieram ao mundo em cumprimento de alguma missão especial ou por intermédio de um desejo divino.

$\checkmark$ Concepção baseada nos pressupostos da normalidade: percebe a deficiência como um desvio da normalidade (modelo médico), devendo ser, portanto, tratada ou cuidada em ambientes especializados ou segregada em áreas específicas da organização, tendo como critério a deficiência.

$\checkmark$ Concepção baseada na inclusão: entende a deficiência como um problema social; a sociedade precisa se adaptar para conviver com a deficiência nos diferentes espaços sociais.

$\checkmark$ Percepção dos benefícios da contratação: corresponde à percepção de que contratar pessoas com deficiência melhora a imagem e o clima da organização.

$\checkmark$ Percepção do vínculo: acredita que pessoas com deficiência são mais comprometidas e estáveis no emprego.

$\checkmark$ Percepção de desempenho: tem como crença que o desempenho e a qualidade do trabalho das pessoas com deficiência são piores em relação às pessoas sem deficiência.

$\checkmark$ Percepção da necessidade de treinamento: ampara-se na necessidade de treinamento como forma de facilitar a inserção e a socialização da PCD no trabalho.

Em síntese, as crenças acerca da deficiência podem refletir na forma como as PCD são recebidas no trabalho. Em análise dos 20 anos de produção acadêmica nacional acerca da inclusão de PCD no mercado de trabalho, Suzano, Nepomuceno, Ávila, Lara e Carvalho-Freitas (2008) concluíram que, frente à coexistência de diferentes formas de ver a deficiência, predo- 
mina ainda o foco no indivíduo, cabendo a ele se adaptar às exigências da sociedade, e não o contrário.

De acordo com a Lei n. 12.470, de 31 de agosto de 2011, considera-se pessoa com deficiência "aquela que tem impedimentos de longo prazo de natureza física, mental, intelectual ou sensorial, os quais, em interação com diversas barreiras, podem obstruir sua participação plena e efetiva na sociedade em igualdade de condições com as demais pessoas" (artigo 20, item 2).

Tal definição parte da perspectiva compartilhada por Finkelstein (1980), de que a pessoa com deficiência não tem apenas uma desvantagem inerente às suas condições de saúde, mas também - e principalmente inerente às condições sociais que dificultam sua disponibilidade de acesso aos direitos comuns a toda população.

Corroborando a ideia de que as atitudes podem servir como obstáculo para a inclusão de PCD no trabalho e visando a contribuir para que tal acesso possa realmente proporcionar dignidade, desenvolvimento e valorização do indivíduo para além de suas limitações, torna-se importante verificar como os profissionais de Recursos Humanos, normalmente responsáveis pela seleção, treinamento e acompanhamento de trabalhadores nas organizações, veem a deficiência e o trabalho das pessoas com deficiência.

Em estudo realizado por Moreira (2011) com PCD inseridas no mercado de trabalho no Brasil e nos Estados Unidos, constatou-se que, no Brasil, há trabalhos específicos para pessoas com deficiência e trabalhos aos quais essas pessoas não têm acesso. Para as PCD, a deficiência é parte constituinte de sua identidade, que só pode ser afirmada mediante sua participação efetiva na sociedade por meio do trabalho. $\mathrm{O}$ não acesso a esse direito é um problema social, visto que, para as pessoas com deficiência, o trabalho é considerado importante para dar sentido às suas vidas (Moreira, 2011).

Uma revisão de literatura realizada por Shur, Kruse e Blank (2005) indica que as atitudes negativas e os estereótipos de empregadores, supervisores e colegas de trabalho formam uma significativa barreira para o emprego de PCD. Para Goss et al. (2000), a ausência de um gerente especializado de $\mathrm{RH}$ pode ser uma variável explicativa para a não-contratação de pessoas com deficiência no Reino Unido. Batavia e Schriner (2005) acreditam que, se a discriminação for elimina- da e as barreiras removidas, as PCD vão ser capazes de funcionar, assim como as pessoas sem deficiência.

Em se tratando das atitudes direcionadas às pessoas com deficiência, Finkelstein (1980) analisa que, na relação entre pessoas com e sem deficiência, o foco está sempre pautado sobre o indivíduo com deficiência, desconsiderando o contexto em que este está inserido. Para o autor, a mudança de foco é decisiva para a mudança de atitude em relação à deficiência. Sendo assim, ele propõe deslocar a questão para o meio social onde a deficiência é definida de modo a reavaliar a forma como ela é vista na sociedade.

Assim, o problema da deficiência passa a ser considerado sob uma nova perspectiva: a partir das relações que se estabelecem com as PCD e a partir das condições que tornam uma pessoa deficiente ou inabilitada, ou seja, que promovam ou restrinjam sua acessibilidade. Para Finkelstein (1980), uma vez que as barreiras sociais são removidas, a deficiência em si é eliminada, pois o foco é deslocado decisivamente para a origem do problema, ou seja, a sociedade inacessível em que a deficiência é criada. De acordo com essa premissa, a inclusão nas empresas deixaria de centrarse apenas na responsabilidade pelo acesso do indivíduo a um cargo e passaria a considerar o compromisso da garantia de respeito e igualdade de tratamento, além de acesso aos meios indispensáveis para que a pessoa com deficiência consiga cumprir com as exigências próprias de sua função.

Nesse ponto, os profissionais de Recursos Humanos têm um importante papel, considerando sua participação efetiva na introdução ou interdição da entrada, acompanhamento e permanência de pessoas com deficiência nas organizações. Para França (2008), dentre as novas perspectivas da administração de Recursos Humanos, está a consciência social acerca da diversidade e inclusão. As PCD necessitam de suporte para se integrarem ao ambiente de trabalho e se desenvolverem como todo e qualquer novo funcionário. No entanto, é dever das organizações desenvolver medidas que rompam com as barreiras necessárias para que as pessoas com deficiência possam realizar seu trabalho com eficácia e qualidade.

Tal proposta torna-se, portanto, relevante, principalmente no que tange aos profissionais de Recursos Humanos, pois estes são normalmente responsáveis pela seleção, treinamento e acompanhamento de trabalhadores nas organizações. Para Suzano (2011), 
mais do que inserir as PCD no ambiente de trabalho por meio da Lei de Cotas, é necessário promover a ressignificação e a reformulação de concepções e valores acerca da pessoa com deficiência, cuja potencialidade pode ser aflorada, desde que lhe sejam oferecidas condições e oportunidades adequadas.

Assim, considera-se que a identificação das crenças e atitudes dos profissionais de Recursos Humanos acerca da deficiência e das possiblidades de trabalho das PCD poderá contribuir para a construção de um conhecimento válido para se tratarem as questões que englobam a inclusão social das PCD, uma vez que são esses profissionais quem implementam as práticas de RH no cotidiano de trabalho das organizações.

\section{MÉTODO}

\section{Participantes}

A pesquisa contou com a participação de 55 profissionais de RH filiados à Associação Brasileira de Recursos Humanos de Minas Gerais (ABRH/MG). Foram enviados 293 convites para participação no estudo, tendo como retorno 59 respostas $(20,1 \%)$. Destas, quatro foram excluídas por não atenderem aos critérios da pesquisa, ficando um total de 55 respostas válidas. Foram consideradas válidas as respostas dos profissionais que afirmaram trabalhar em empresas ${ }^{2}$ com mais de 100 funcionários (número mínimo estipulado pela Lei de Cotas), bem como aqueles que, mesmo trabalhando em empresas com menos de 100 funcionários, afirmaram ter, em seu quadro de efetivos, pessoas com deficiência (critério utilizado para inclusão na pesquisa).

Dos respondentes, $20 \%$ foram pessoas do sexo masculino e $80 \%$ do sexo feminino. De acordo com a faixa etária, 43,6\% encontram-se na faixa dos 21 aos 30 anos e $34,5 \%$ entre 31 e 40 anos. Quanto à religião $^{3}$, a maioria é católica $(63,6 \%)$, seguida de espírita $(16,4 \%)$ e protestante $(7,3 \%)$. Em relação à formação dos respondentes, $25,4 \%$ possuem graduação em Psicologia e mais de $30 \%$ em cursos como Administração e Pedagogia, dentre outros. Já 61,8\% têm especialização, sendo a maioria $(36,3 \%)$ especialista em Gestão de Pessoas. Analista de RH é o cargo ocupado por $36,4 \%$ dos respondentes, enquanto $27,3 \%$ são gerentes de Recursos Humanos, o que pressupõe, nesse último cargo, maior autonomia para tomar decisões sobre políticas inclusivas a serem adotadas. De acordo com o tempo no cargo, 43,6\% ocupam o cargo há mais de cinco anos e 7,2\% há menos de um ano.

Em relação às empresas em que trabalham os profissionais de RH pesquisados, é possível constatar que $38,1 \%$ delas têm menos de dez funcionários com deficiência contratados. Quanto ao tipo de experiência prévia com PCD (se positiva ou negativa), $81,1 \%$ dos respondentes afirmaram ter sido positiva, $1,8 \%$ negativa e $14,5 \%$ disseram não ter tido contato prévio com PCD.

\section{Instrumentos e Medidas}

A pesquisa compreendeu a aplicação de três questionários, um inventário e entrevista estruturada. Num primeiro momento, visando a coletar dados acerca do perfil do respondente foi aplicado o questionário Caracterização do Respondente. Em seguida, foi aplicado o Questionário Práticas de RH em Relação à Inclusão de Pessoas com Deficiência no Trabalho, elaborado pela pesquisadora e que continha questões acerca das práticas de RH realizadas no recrutamento, seleção, treinamento e desenvolvimento de PCD nas empresas.

Para verificar as concepções de deficiência dos profissionais de Recursos Humanos, foi aplicado o Inventário de Concepções de Deficiência em Situações de Trabalho (ICD-ST) validado por Carvalho-Freitas (2012). O inventário é um instrumento de diagnóstico constituído de questões fechadas, que caracterizam as principais interpretações de deficiência compartilhadas e existentes ao longo da história.

Por fim, o Questionário Atitude Frente às Possibilidades de Ação para a Inclusão de Pessoas com Deficiência no Trabalho, adaptado do Inventário de Ações Possíveis no Processo de Inserção de PCD (IAP) e validado por Carvalho-Freitas, Souto, Simas, Costa e Santos (2012), foi utilizado com o intuito de verificar as ações consideradas efetivamente possíveis de serem realizadas. Os instrumentos aplicados, com exceção do Questionário de Caracterização do Respondente, são compostos por Escala Likert de escolha forçada, com opções que variam de 1 (discordo totalmente) a 6 (concordo totalmente).

As entrevistas realizadas sob a perspectiva qualitativa tiveram como objetivo investigar em profundidade as informações coletadas de modo a contemplar questões que porventura não constaram no questionário. 


\section{Procedimentos}

A coleta de dados foi realizada inicialmente por meio de contato telefônico com a ABRH/MG, para solicitar o acesso aos e-mails dos associados. De posse desses contatos, uma mensagem nominal, convidando para participação na pesquisa, foi enviada a todos os associados, com um link de acesso aos questionários, perfazendo um total de 293 mensagens enviadas.

A aplicação dos questionários se deu por autopreenchimento, por meio eletrônico, em que era possível visualizar uma breve apresentação da pesquisa, os contatos da instituição de ensino e das pesquisadoras, bem como as devidas instruções para o preenchimento dos questionários. Em seguida, a leitura do Termo de Consentimento Livre e Esclarecido direcionava o participante a realizar o aceite para participação na pesquisa.

O prazo dado para a devolução das respostas foi de 30 dias, sendo prorrogado por mais 30 em razão da baixa taxa de respostas obtida nas primeiras tentativas. Contudo, após o período estipulado, foram recebidas 59 respostas, sendo que, desse total, 55 foram consideradas válidas, correspondendo a uma taxa de 18,7\%.

Para complementar as informações colhidas por meio dos questionários, foi enviado um roteiro de entrevista composto de nove questões abertas aos 55 respondentes da primeira etapa, tendo como retorno uma taxa de respostas de $12,7 \%$ (sete respostas).

\section{Análise de Dados}

Os dados quantitativos, obtidos por meio dos questionários, foram analisados pelo programa SPSS for Windows (Statistical Package for Social Sciences) mediante as seguintes técnicas estatísticas:

$\checkmark$ Estatística descritiva: análise da distribuição percentual das respostas quanto à caracterização dos respondentes.

$\checkmark$ Teste de qui-quadrado: para verificar a independência entre fatores, ou seja, se as práticas de RH em relação à inclusão de PCD no trabalho, concepções de deficiência, bem como as atitudes frente às possibilidades de ação para inclusão de pessoas com deficiência nas organizações dependem de fatores como religião, grau de escolaridade, cargo, tipo de deficiência e experiência prévia com PCD.

$\checkmark$ Análise de correlação de Spearman: para verificar a ocorrência de relações (direção e intensi- dade) entre as variáveis concepções de deficiência dos profissionais de Recursos Humanos, práticas de RH com relação à inclusão de PCD no trabalho e atitudes frente às possibilidades de ação para inclusão de pessoas com deficiência nas organizações. A opção pela correlação de Spearman justifica-se em razão do número de respondentes da pesquisa.

$\checkmark$ Análise de Cluster: visando verificar se os respondentes poderiam ser agrupados segundo os fatores analisados: crenças (concepções de deficiência), atitudes em relação às ações possíveis para a inserção de PCD e práticas de RH implementadas pelas organizações. O método foi o de Ward, baseado nos princípios da análise de variância. A medida de similaridade utilizada foi a distância euclidiana, que é representada pela proximidade entre observações ao longo das variáveis na estatística de agrupamento. Para determinar o número de conglomerados, utilizou-se o gráfico dos passos de agrupamentos em relação ao nível de distância. Detectou-se a formação de três conglomerados. Esses clusters visavam identificar padrões de atitudes em relação ao trabalho com PCD.

\section{RESULTADOS}

No que diz respeito às concepções de deficiência, a maioria dos profissionais de Recursos Humanos discorda da concepção espiritual da deficiência, ou seja, não concorda que a pessoa com deficiência esteja no mundo para cumprir algum tipo de missão divina ou vontade sobrenatural. Entretanto, esse pressuposto ainda permeia a dúvida de $13,5 \%$ dos profissionais de $\mathrm{RH}$, o que significa que ainda existe, mesmo que em menor proporção, o pensamento de que a PCD seja especial e deva ser tratada com cuidado e proteção ou até mesmo com caridade.

Há dúvidas entre $51 \%$ dos respondentes quanto à concepção baseada na normalidade. Tal crença implica considerar mais as limitações da pessoa com deficiência do que suas reais possibilidades. As pessoas que tendem a esse tipo de concepção acreditam que as PCD são inadequadas para alguns cargos e locais, sendo, muitas vezes, segregadas em espaços delimitados dentro da própria organização.

A concepção baseada nos pressupostos da inclusão prevalece entre $54,5 \%$ dos profissionais de $\mathrm{RH}$, o que confirma uma tendência atual em considerar as diver- 
sas formas de promover a acessibilidade a todas as pessoas (Carvalho-Freitas, 2009; Carvalho-Freitas e Marques, 2009, 2010; Suzano, 2011; Assis, 2012). Essa percepção dos profissionais de Recursos Humanos de que as pessoas com deficiência podem desempenhar qualquer tipo de trabalho (desde que lhes sejam oferecidas condições adequadas) é importante para impulsionar gestores e colegas de trabalho quanto aos objetivos da inclusão.

Finalmente, quanto à matriz técnica (desempenho, vínculo, benefícios da contratação e necessidade de treinamento), $89 \%$ dos profissionais de $\mathrm{RH}$ acreditam que não há diferença entre um funcionário com e sem deficiência nos quesitos desempenho, produtividade $\mathrm{e}$ qualidade do trabalho. Além disso, 41,8\% discordam da ideia de que as pessoas com deficiência se sentem mais vinculadas ao trabalho e são mais estáveis e comprometidas do que as pessoas sem deficiência. Em contrapartida, $34,6 \%$ concordam que a contratação de PCD melhora o clima e as imagens (interna e externa) da empresa, outros $34,6 \%$ têm dúvidas sobre isso e, ainda, $76,4 \%$ concordam que é necessário o treinamento de toda a equipe para facilitar a inserção e a socialização das PCD.

Levando-se em conta o que é praticado nas organizações e não a opinião pessoal dos profissionais de Recursos Humanos, apenas 14\% dos respondentes afirmam existirem práticas de $\mathrm{RH}$ inclusivas com relação à entrada de PCD nas empresas, o que indica uma forte tendência a haver apenas a inserção da pessoa, e não sua efetiva inclusão no trabalho. Além disso, $60 \%$ concordam que a Lei de Cotas é difícil de ser cumprida, alegando, dentre vários motivos, falta de capacitação das PCD, altos gastos com as adaptações necessárias e falta de incentivos do governo para que as empresas possam cumprir a Lei. Esses dados indicam que pouco tem sido feito para mudar a realidade das pessoas com deficiência, as quais, mesmo tendo o respaldo da Lei de Cotas, ainda necessitam vencer vários obstáculos para se manterem no mercado de trabalho.

Para verificar se as práticas de $\mathrm{RH}$ em relação à inclusão de PCD no trabalho e as concepções de deficiência dependiam de fatores como religião, cargo, tipo de deficiência e experiência prévia com PCD, foi utilizado o teste de qui-quadrado.

Constatou-se que, dentre os fatores analisados, dois se apresentaram, significativamente, como dependentes da experiência prévia com PCD (positiva ou negativa): as práticas de RH com relação à entrada de PCD nas organizações $(\chi 2=71,165, p=0,000)$ e as práticas de RH com relação às adequações necessárias para o trabalho com $\operatorname{PCD}(\chi 2=58,405, p=0,030)$. Isso indica que o tipo de experiência prévia com funcionários com deficiência pode influenciar as práticas organizacionais com relação à entrada e permanência dessas pessoas nas empresas.

Testifica-se também que $71 \%$ dos profissionais de Recursos Humanos acreditam nas possibilidades de ação para inclusão de PCD nas organizações, mas $29 \%$ dos respondentes ainda têm dúvidas sobre tais possibilidades.

A Tabela 1 apresenta os resultados da análise correlacional de Spearman entre os fatores do Inventário de Concepções de Deficiência em Situações de Trabalho (ICD-ST), as possibilidades de ação para inclusão de PCD nas empresas e as práticas de RH com relação às adequações no ambiente de trabalho.

Tabela 1

Valores de Correlação de Spearman entre os Fatores do ICD-ST e Demais Construtos

\begin{tabular}{lcc}
\multicolumn{1}{c}{ Concepção de Deficiência } & $\begin{array}{c}\text { Possibilidades de ação } \\
\text { para inclusão }\end{array}$ & $\begin{array}{c}\text { PRH Adequações no ambiente de } \\
\text { trabalho }\end{array}$ \\
Fator benefícios da contratação & $0,323^{*}$ & - \\
Fator vínculo & - & - \\
Concepção baseada na normalidade & - & - \\
Concepção baseada na inclusão & $0,587^{* *}$ & - \\
Fator desempenho & $-0,506^{* *}$ & - \\
Concepção espiritual & $0,325^{*}$ & $-0,273^{*}$ \\
\hline
\end{tabular}

Notas: ${ }^{*} p<0,05 ;{ }^{* *} p<0,01$. 
Os resultados do teste rho de Spearman indicam que os fatores do ICD-ST tiveram relacionamentos significativos do ponto de vista estatístico, indicando que:

quanto maior a crença nos pressupostos da inclusão, da matriz espiritual e dos benefícios da contratação, maior a percepção sobre as possibilidades de ação para inclusão de PCD nas empresas (atitude mais favorável). Em contrapartida:

quanto mais se acredita no fator desempenho, menor a percepção sobre as possibilidades de ação para inclusão; ou seja, quanto mais se acredita que o desempenho da pessoa com deficiência é ruim em relação às demais pessoas, menos se acredita que é possível a realização de ações voltadas para a sua inclusão;

quanto mais os profissionais de Recursos $\mathrm{Hu}-$ manos percebem que existem na empresa práticas de RH voltadas para adequações no ambien- te de trabalho, menor a percepção de necessidade de treinamento; ou seja, se o ambiente de trabalho já está adequado para as $\mathrm{PCD}$, os treinamentos tornam-se desnecessários;

$\checkmark$ não foram encontradas relações entre as práticas de RH voltadas para a entrada de PCD no trabalho e as concepções de deficiência;

não foram encontradas relações entre as concepções de deficiência compartilhadas pelos profissionais de Recursos Humanos e a percepção de dificuldades com relação à Lei de Cotas e à permanência de pessoas com deficiência nas empresas.

Foi feita também análise correlacional entre as concepções de deficiência e fatores como crença na adequação da Lei de Cotas e dificuldades quanto à falta de conhecimento das pessoas em lidar com PCD, identificados no questionário de Caracterização do Respondente. A Tabela 2 apresenta os resultados:

Tabela 2

Valores de Correlação de Spearman entre Concepções de Deficiência e Percepção de Dificuldades no Trabalho com PCD

\begin{tabular}{|c|c|c|c|c|c|}
\hline $\begin{array}{c}\text { Concepção de } \\
\text { Deficiência }\end{array}$ & $\begin{array}{c}\text { Acho adequado o } \\
\text { Brasil ter Lei de Cotas } \\
\text { para o trabalho com } \\
\text { PCD }\end{array}$ & $\begin{array}{c}\text { Acho que a falta } \\
\text { de conhecimento } \\
\text { sobre como tornar } \\
\text { os espaços físicos } \\
\text { acessíveis dificulta } \\
\text { o trabalho com } \\
\text { PCD }\end{array}$ & $\begin{array}{l}\text { Acho que a } \\
\text { falta de } \\
\text { conhecimento } \\
\text { das pessoas } \\
\text { dificulta o } \\
\text { trabalho com } \\
\text { PCD }\end{array}$ & $\begin{array}{l}\text { Acho que a } \\
\text { falta de } \\
\text { convivência } \\
\text { com PCD } \\
\text { dificulta o } \\
\text { trabalho } \\
\text { com estas }\end{array}$ & $\begin{array}{c}\text { Acho que a } \\
\text { falta de } \\
\text { conhecimento } \\
\text { sobre } \\
\text { recursos } \\
\text { materiais e } \\
\text { tecnológicos } \\
\text { dificulta o } \\
\text { trabalho com } \\
\text { PCD }\end{array}$ \\
\hline Fator benefícios da contratação & - & - & $0,417 * *$ & $0,300 *$ & $0,388 * *$ \\
\hline Fator vínculo & - & - & - & - & - \\
\hline $\begin{array}{l}\text { Concepção baseada na } \\
\text { normalidade }\end{array}$ & $-0,309 *$ & - & - & - & - \\
\hline Concepção espiritual & - & - & - & - & - \\
\hline $\begin{array}{l}\text { Percepção de necessidade de } \\
\text { treinamento }\end{array}$ & - & $0,282 *$ & $0,369 * *$ & $0,399 * *$ & $0,362 * *$ \\
\hline
\end{tabular}

Notas: ${ }^{*} p<0,05 ; * * p<0,01$. 
Essas correlações indicam associação entre os seguintes construtos estudados:

Quanto maior a crença nos benefícios da contratação (que se refere aos benefícios em relação à imagem da empresa e ao clima interno), maior a percepção de que falta conhecimento das pessoas sobre a deficiência, conhecimento em relação aos recursos materiais e tecnológicos, bem como falta convivência com as PCD. A partir dessa correlação, levanta-se a hipótese de que é possível que os profissionais que mais acreditem nos benefícios da contratação são aqueles que mais identificam os problemas relacionados aos conhecimentos da empresa sobre a deficiência e sobre os recursos para a inclusão, além da falta de convivência prévia como possíveis dificultadores para uma efetiva inclusão das pessoas com deficiência.

Quanto mais se compartilha dos pressupostos da normalidade (deficiência como desvio da normalidade), menos se acha adequada a existência da Lei de Cotas no Brasil para o trabalho com PCD. Essa correlação indica a importância de se identificar e trabalhar essa concepção de deficiência entre os profissionais de Recursos Humanos, pois ela pode ser um dificultador para implementar a inclusão de pessoas com deficiência nas organizações.

Quanto mais se acredita na concepção baseada na inclusão (percepção de que as PCD podem desempenhar adequadamente o trabalho desde que sejam realizadas as adaptações nas condições e instrumentos de trabalho), maior a percepção de que falta conhecimento das pessoas sobre a deficiência e sobre recursos materiais e tecnológicos que podem auxiliar na inclusão. A hipótese que se levanta é que os profissionais que compartilham os pressupostos da inclusão são aqueles que conseguem identificar os problemas relacionados aos conhecimentos das pessoas como relevantes para a inclusão de pessoas com deficiência.

Quanto maior a crença no desempenho (definido como negativo em relação às demais pessoas), maior a percepção de que a Lei de Cotas é adequada para garantir trabalho às PCD (hipótese de que, por esse motivo - desempenho pior -, sem as cotas, as pessoas com deficiência não entrariam no mercado de trabalho) e menos se acredita que falta conhecimento das pessoas (sobre a acessibilidade e existência de recursos). Esses profissionais acreditam que o maior problema é o desempenho das $\mathrm{PCD}$, e não as condições ou conhecimentos que se tenha.

$\checkmark$ Quanto maior a percepção da necessidade de treinamento, mais se acredita que falta conhecimento sobre a deficiência, convivência com PCD e conhecimentos sobre acessibilidade e recursos para a inclusão.

Finalmente, para dividir o grupo de respondentes por similaridade, pelo agrupamento de alguns fatores, utilizou-se a análise de Cluster, objetivando melhor delimitação sobre os perfis dos profissionais de Recursos Humanos participantes desta pesquisa. Aplicou-se a análise de Cluster no conjunto dos seis fatores do Inventário de Concepções de Deficiência em Situações de Trabalho (ICD-ST) e, depois, nos demais fatores relacionados à inclusão de PCD no trabalho.

Cluster 1: Perfil menos favorável à inclusão. Neste Cluster, foram agrupados dez respondentes. Este grupo tem como principais características que o distingue dos demais o fato de ser composto por profissionais de RH que pertencem a empresas que possivelmente têm algumas práticas de responsabilidade social e de sensibilização, que têm dúvida se a Lei de Cotas seja uma ação da empresa apenas para cumprimento da legislação e que concordam pouco que tenham dificuldades para encontrar pessoas com deficiência qualificadas no mercado de trabalho. Por outro lado, são os profissionais que menos concordam (concordam pouco) que seja possível a realização de ações que contribuam efetivamente para a inserção de PCD e que menos concordam com os pressupostos da inclusão. Isto é, que, em se garantindo as condições e instrumentos de trabalho adequados, as pessoas com deficiência podem desempenhar adequadamente seu trabalho. Ressalta-se que, neste grupo, prevalecem pessoas com nível de escolaridade correspondente ao ensino superior completo. Pelo menos cinco respondentes deste grupo trabalham com mais de dez pessoas com deficiência na organização. Possivelmente, é um grupo que pouco contribuiria para a implementação de políticas de diversidade na organização. 
Cluster 2: Perfil mais favorável à inclusão. A maioria dos respondentes foi agrupada neste Cluster, perfazendo um total de 25 pessoas. É um grupo formado por profissionais que consideram que a empresa em que trabalham tem algumas estratégias de responsabilidade social e de sensibilização para a entrada de PCD, além de ter uma tendência a concordar que a Lei de Cotas tem sido apenas uma ação de cumprimento da legislação e que tem dificuldades para encontrar PCD com a formação necessária para o trabalho. Por outro lado, é o grupo em que as pessoas mais acreditam que seja possível a realização de ações para garantir a inserção de pessoas com deficiência, que mais concorda que a inserção de PCD possa beneficiar a imagem e o clima da organização, que mais concorda que, em se modificando as condições e instrumentos de trabalho, as pessoas com deficiência podem desempenhar adequadamente seu trabalho (matriz da inclusão) e que mais discorda que o desempenho das PCD seja pior que o das demais pessoas. Prevaleceu a especialização como principal nível de escolaridade deste grupo. Dentre os 25 respondentes deste grupo, oito trabalham com mais de dez pessoas com deficiência na organização. Considera-se que este grupo tenha um padrão de crenças e atitudes mais favorável à inclusão, embora reconheçam as dificuldades desse processo, sendo formado por pessoas que podem contribuir mais efetivamente para a mudança dessa realidade (caso seja possível em função do poder que elas exerçam nas organizações em que trabalham).

Cluster 3: Perfil com foco na deficiência. Este Cluster corresponde a 20 respondentes. Este grupo é formado por profissionais que consideram as empresas em que trabalham como as que menos têm políticas definidas de responsabilidade social e estratégias de sensibilização para a entrada de PCD comparativamente com os outros dois agrupamentos. Além disso, é o grupo que mais concorda que as empresas em que trabalham inserem pessoas com deficiência apenas para atender à Lei de Cotas e que mais afirma ter dificuldades para encontrar PCD com formação adequada para o trabalho. Por outro lado, é um grupo que concorda pouco que seja possível o desenvolvimento de ações que garantam a inserção de PCD no trabalho, que mais concorda com os pressupostos da normalidade, isto é, que foca a deficiência e que acredita que apenas alguns setores são adequados ao trabalho dessas pessoas. Também é o grupo que mais concorda que são necessários treinamentos para garantir a inserção de pessoas com deficiência. A especialização foi o nível de escolaridade predominante neste grupo. Aqui, sete pessoas trabalham com mais de dez PCD na organização. A hipótese que se levanta é que, como o grupo tem por foco a deficiência, a perspectiva de treinar para trabalhar com PCD seja uma ação privilegiada para conhecer as deficiências e saber como trabalhar com elas, ao invés de se pensar a deficiência como uma dimensão social e política que divide homens, sendo necessárias ações que viabilizem o pleno exercício do direito por parte de todos e efetivas mudanças nas condições de acesso dessas pessoas (Barnes, 1999; Barnes, Oliver, \& Barton, 2002).

Foi realizado o teste de qui-quadrado com o objetivo de se verificar se os clusters estão relacionados com algumas variáveis de caracterização dos respondentes. Para a variável grau de escolaridade, o resultado do teste de qui-quadrado indica uma dependência $(\chi 2=22,194$, com 8 graus de liberdade e valor de $p=$ $0,005)$; ou seja, estar em um ou outro agrupamento depende do grau de escolaridade. No primeiro cluster, ficou a maioria de respondentes com escolaridade entre ensino médio e superior completo; no segundo cluster, os que tinham entre ensino superior completo e especialização; e, no terceiro, os respondentes que tinham entre especialização e mestrado. Não foram observadas outras relações entre os clusters e demais fatores de caracterização dos respondentes. Esse resultado indica a necessidade de se pesquisarem as abordagens sobre a inclusão de PCD que têm sido trabalhadas nas especializações no País.

\section{DISCUSSÃO}

O presente estudo teve como objetivo verificar a existência de relação entre crenças, atitudes e práticas dos profissionais de $\mathrm{RH}$ em relação à inclusão de pessoas com deficiência nas organizações. De forma geral, constatou-se que a utilização dos construtos concepções de deficiência, ações consideradas possíveis para garantir a inclusão de pessoas com deficiência no trabalho e percepção das práticas de RH utilizadas nas organizações contribuíram para a identificação de posições diferenciadas dos profissionais de Recursos Humanos frente à inclusão, conforme identificado nas análises de correlação e clusters.

Foi verificado que, em relação às concepções de deficiência, os profissionais de RH não têm uma posição clara que possa efetivamente ter potencial para se 
transformar em ações, pois pouco mais da metade deles concordam com os pressupostos da inclusão; em contrapartida, mais de $50 \%$ também têm dúvidas se a deficiência não seria um desvio da normalidade e que apenas alguns setores seriam adequados a elas. Também têm posições divergentes quanto aos benefícios da contratação dessas pessoas para as empresas. Essa dubiedade em relação às concepções de deficiência fragiliza a construção de ações, pois são permeadas por dúvidas sobre pressupostos importantes para o trabalho com essas pessoas.

Com isso, as práticas de $\mathrm{RH}$ para receber e manter pessoas com deficiência nas empresas acabam por refletir tais dúvidas. Nota-se que, na tentativa de englobar num único processo pessoas com e sem deficiência, ainda é evidente a tendência em desconsiderar as necessidades diferenciadas das PCD. A não modificação das práticas de RH é, contudo, justificada pelo discurso da igualdade e pelo tratamento sem privilégios e diferenças. Dessa forma, de acordo com as entrevistas, percebe-se que poucas têm sido as mudanças evidenciadas nas práticas de RH mantidas pela maioria das empresas, destacando-se apenas algumas adaptações físicas e estruturais, quando necessário. Conclui-se que tais práticas visam apenas ao cumprimento de normas, não podendo ser consideradas efetivamente inclusivas.

Também foi observado que, quando existem, as práticas de RH com relação à entrada de $\mathrm{PCD}$, bem como as adequações nas condições de trabalho dependem do tipo de experiência prévia com pessoas com deficiência. Pela análise de Cluster, foi possível identificar que o grupo com maior disposição para o trabalho com PCD é o de pessoas que trabalham com mais de dez pessoas com deficiência no ambiente de trabalho, além de ser o grupo que mais compartilha os pressupostos da concepção de inclusão e que acredita que sejam possíveis as ações de modificações das condições de trabalho. Esse resultado reafirma a associação entre as crenças sobre determinado objeto e as crenças acerca do grau de facilidade ou dificuldade em executar uma determinada ação (crenças sobre controle) identificada em diversas pesquisas (Ajzen, 1985, 1988, 1991). Além disso, constata que pessoas com especialização relacionada à Gestão de Pessoas são mais favoráveis ao trabalho com PCD.

Ainda em relação às práticas de $\mathrm{RH}$, verificou-se que não há relação entre concepções de deficiência e práticas de $\mathrm{RH}$ inclusivas, ou seja, a forma como a deficiência é vista por um profissional de Recursos Humanos (sua crença ou concepção de deficiência) não tem reflexo nas práticas de RH mantidas na empresa para a inclusão de PCD. Tal achado é importante para que se possa refletir sobre o papel desse sujeito (profissional de RH) como facilitador de mudanças nas empresas, promovendo uma reavaliação de sua atuação.

Também foi evidenciado que os profissionais de Recursos Humanos podem ser agrupados em três diferentes tipos de perfis quanto à sua atitude (mais ou menos favorável) para o trabalho com PCD: o perfil menos favorável à inclusão, o perfil mais favorável à inclusão e o perfil com foco na deficiência. Do primeiro grupo, pouco se pode esperar em termos de contribuição para a implementação de políticas de diversidade nas organizações. $\mathrm{O}$ segundo grupo pode contribuir mais efetivamente para essa realidade e o terceiro grupo visa apenas ao cumprimento da Lei de Cotas. Nota-se que, devido a não haver uma ampla adoção dos princípios da inclusão pelos profissionais de RH, a simples inserção de pessoas com deficiência nas empresas ainda é um problema que merece atenção, pois é sabido que o simples acesso garantido pela Lei de Cotas não pressupõe a garantia de igualdade às diversas oportunidades oferecidas aos trabalhadores.

Finalmente, os resultados da pesquisa mostram que, mesmo que a concepção de deficiência dos profissionais de Recursos Humanos seja favorável aos preceitos da inclusão e mesmo que esses profissionais acreditem nas possibilidades de ações que viabilizem esse intento, ainda assim tendem a justificar a falta de práticas e ações inclusivas de $\mathrm{RH}$ nas dificuldades percebidas, sejam elas em relação ao cumprimento da Lei de Cotas, nas dificuldades relativas à permanência de PCD no trabalho ou mesmo na falta de adequações da empresa.

Cabe ressaltar que a estratégia de muitas empresas para a não contratação de pessoas com deficiência é amparar-se nas dificuldades mencionadas (Ribeiro \& Carneiro, 2009). No entanto, tais dificuldades precisam ser encaradas como desafios a serem superados. A figura do profissional de RH nas empresas pode influenciar gestores e chefias acerca dos benefícios do adequado gerenciamento da diversidade pela inclusão nas organizações. 


\section{CONSIDERAÇÕES FINAIS}

Constata-se por meio desta pesquisa que a inclusão demanda modificações profundas na cultura de uma empresa. Sabe-se que não é fácil quebrar antigos paradigmas que consideram as limitações da pessoa com deficiência em detrimento de suas potencialidades. Mas exemplos cotidianos nos mostram que elas podem, desde que lhes sejam oferecidas condições adequadas, realizar suas atividades com qualidade, assim como as pessoas sem deficiência.

A atitude de incluir PCD nas empresas, e não apenas inseri-las, deve ser encarada como responsabilidade social, devendo ser afirmada nas políticas e diretrizes da organização (Ribas, 2007; Vitor, 2011). No entanto, cabe ao profissional de Recursos Humanos o desafio de buscar estratégias que promovam o empenho de todos para que as PCD sejam tratadas com respeito e dignidade, sendo consideradas como iguais colaboradoras do processo produtivo das empresas.

Como limitação importante desta pesquisa, destaca-se o tamanho da amostra e sua distribuição apenas pelo estado de Minas Gerais. O reduzido número de respondentes não permite generalizações, devendo-se considerar os resultados encontrados apenas para a população em questão. Outra limitação se refere ao fato de terem sido realizadas entrevistas nãopresenciais, o que impossibilita a rica troca entre entrevistador e entrevistado.

Outro limite da pesquisa foi não ter incluído os motivos que, para os respondentes, caracterizariam o que significava para eles experiência prévia positiva ou negativa, uma vez que essa questão foi identificada como relevante na explicação das práticas de RH referentes à entrada de pessoas com deficiência e em relação às adaptações das condições de trabalho. Novas pesquisas visando a identificar o que caracteriza para os profissionais de $\mathrm{RH}$ experiências prévias positivas e negativas deverão ser realizadas, objetivando ampliar o conhecimento sobre essa temática e auxiliar no processo de inclusão dessas pessoas.

A presente pesquisa não tem a pretensão de abranger todas as questões envolvidas no processo de inclusão de pessoas com deficiência no trabalho. No entanto, considera o profissional de Recursos Humanos um importante ator no processo de inclusão.

\section{REFERÊNCIAS}

Ajzen, I. (1985). From intentions to actions: A theory of planned behavior. In J. Kuhi, \& J. Beckmann (Eds.). Action control: From cognition to behavior (pp. 11-39). Heidelberg: Springer.

Ajzen, I. (1988). Attitudes, personality and behavior. England: Open University Press.

Ajzen, I. (1991). The theory of planned behavior. Organizational Behavior and Human Decision Processes, 50, 179-211.

Aranha, M. S. F. (1995). Integração social do deficiente: análise conceitual e metodologia. Temas em Psicologia, 2, 63-70.

Assis, A. M. (2012). A socialização de pessoas com deficiência: Um estudo de caso numa organização de grande porte. Dissertação de mestrado, Universidade Federal de São João del-Rei, São João del-Rei, MG, Brasil.

Barnes, C. (1999). Disability Studies: New or not so new directions? Disability \& Society, 14(4), 577-580.

Barnes, C., Oliver, M., \& Barton, L. (2002). Disability studies today. Cambridge: Polity.

Batavia, A. J., \& Schriner, K. (2005). The Americans with Disabilities Act as engine of social change: Models of disability and the potential of a civil rights. Policy Studies Journal, 4(29), 690-702.

Blanchard, K. L. (2001). Attitudes of employers toward people with disabilities: A comparison of Berlin, Germany, and Milwaukee, Wisconsin, USA. The Graduate College. University of Wisconsin-Stout, EUA.

Carvalho-Freitas, M. N. (2007). A inserção de pessoas com deficiência em empresas brasileiras. Um estudo sobre as relações entre concepções de deficiência, condições de trabalho e qualidade de vida no trabalho. Tese de doutorado, Universidade Federal de Minas Gerais, Belo Horizonte, MG, Brasil.

Carvalho-Freitas, M. N. (2009). Inserção e gestão do trabalho de pessoas com deficiência: Um estudo de caso. $R A C$, 13(Edição Especial), 121-138.

Carvalho-Freitas, M. N. (2012). Validação do inventário de concepções de deficiência em situações de trabalho (ICD-ST). Psico-USF, 17(1), 33-42.

Carvalho-Freitas, M. N., \& Marques, A. L. (2007). A diversidade através da História: A inserção no trabalho de pessoas com deficiência. Organizações \& Sociedade, 14(41), 59-78.

Carvalho-Freitas, M. N., \& Marques, A. L. (2009). Pessoas com deficiência e Trabalho: Percepção de gerentes e pós-graduandos em administração. Psicologia, Ciência e Profissão, 29(2), 244-257. 
Carvalho-Freitas, M. N., \& Marques, A. L. (2010). Formas de ver as pessoas com deficiência: Um estudo empírico do construto de concepções de deficiência em situações de trabalho. Rev. Adm. Mackenzie, 11(3), 100-129.

Carvalho-Freitas, M. N., Souto, J. F., Simas, A. L. B., Costa, N. B., \& Santos, L. M. M. (2012). Trabalhar com pessoas com deficiência: Futuros profissionais têm essa disposição? XXXVI Encontro da Anpad. Rio de Janeiro: Enanpad.

Finkelstein, V. (1980). Attitudes and Disabled People: Issues for Discussion. New York, USA: International Exchange of Information in Rehabilitation.

França, A. C. L. (2008). Práticas de recursos humanos. PRH. Conceitos, Ferramentas e Procedimentos. São Paulo: Editora Atlas.

Garcia, V. G. (2014). Panorama da inclusão das pessoas com deficiência no mercado de trabalho no Brasil. Trab. Educ. Saúde [online], 12(1), 165-187.

Goss, D., Goss, F., \& Adam-Smith, D. (2000). Disability and employment: A comparative critique of UK legislation. The International Journal of Human Resource Management, 11(4), 807-821.

Instituto Brasileiro de Geografia e Estatística (IBGE). (2010). Censo 2010. Recuperado em 5 novembro, 2011, de http://www.censo2010.ibge.gov.br

Instituto Ethos de Empresas e Responsabilidade Social. (2010). Perfil social, racial e de gênero das 500 maiores empresas do Brasil e suas ações afirmativas: pesquisa 2010. São Paulo/SP.

Lei n. 8.213, de 24 de julho de 1991. (1991). Dispõe sobre os Planos de Benefícios da Previdência Social, e dá outras providências. Brasília. 2000. Recuperado em 2 novembro, 2011, de www.planalto.gov.br/ccivil_03/Leis/ L8213cons.htm

Lei n. 12.470, de 31 de agosto de 2011. (2011). Altera os arts. 20 e 21 e acrescenta o art. 21-A à Lei n. 8.742, de 7 de dezembro de 1993 - Lei Orgânica de Assistência Social, para alterar regras do benefício de prestação continuada da pessoa com deficiência. Recuperado em 28 fevereiro, 2012, de http://www.planalto.gov.br/ccivil_03/_ Ato2011-2014/2011/Lei/L12470.htm

Lima, F. J., Guedes, L. C., \& Guedes, M. C. (2010). Áudiodescrição: Orientações para uma prática sem barreiras atitudinais. Revista Brasileira de Tradução Visual, 2. Revista Eletrônica. Recuperado em $1^{\circ}$ novembro, 2011, de http://www.rbtv.associadosdainclusao.com.br/index. php/principal/article/viewArticle/28

Ministério do Trabalho e Emprego (MTE) (2011). Características do emprego formal segundo a Relação Anual de Informações Sociais. Recuperado em 18 dezembro, 2012, de http://www.mte.gov.br/rais/2011/arquivos/ Resultados_Definitivos.pdf
Moreira, L. B. (2011). Socialização organizacional e dinâmica identitária de pessoas com deficiência: Um estudo no Brasil e nos Estados Unidos. Dissertação de mestrado, Universidade Federal de Lavras, Lavras, MG, Brasil.

Oliveira, M. A., Goulart Jr., E., \& Fernandes, J. M. (2009). Considerações sobre políticas públicas nos Estados Unidos, União Europeia e Brasil. Revista Brasileira de Educação Especial, 15(2), 219-232.

Portaria n. 2.344, de 3 de novembro de 2010. (2010). Faz publicar a Resolução $\mathrm{n}^{\circ}$ 01, de 15 de outubro de 2010, do Conselho Nacional dos Direitos da Pessoa Portadora de Deficiência - CONADE, que altera dispositivos da Resolução $\mathrm{n}^{\circ} 35$, de 06 de julho de 2005, que dispõe sobre seu Regimento Interno.

Pessotti, I. (1984). Deficiência mental: Da superstição à ciência. Biblioteca de Psicologia e Psicanálise (Vol. 4). São Paulo: Editora da Universidade de São Paulo.

Ribas, J. (2007). Preconceito contra as pessoas com deficiência. São Paulo: Editora Cortês.

Ribeiro, M. A., \& Carneiro, R. (2009). A inclusão indesejada: As empresas brasileiras face à Lei de Cotas para pessoas com deficiência no mercado de trabalho. $O \& S O r$ ganização e Sociedade (UFBA), 16(50), 545-564.

Sassaki, R. (2006). Inclusão: Construindo uma sociedade para todos $\left(7^{\mathrm{a}}\right.$ ed.). Rio de Janeiro: WVA.

Shur, L., Kruse, D., \& Blank, P. (2005). Corporate culture and the employment of persons with disabilities. Behavioral Sciences and the Law, 23, 3-20.

Suzano, J. C. C. (2011). Concepções de deficiência e percepções de desempenho por tipo de deficiência: A perspectiva dos gestores. Dissertação de mestrado, Programa de Pós-graduação em Psicologia, Universidade Federal de São João del-Rei, São João del-Rei, MG, Brasil.

Suzano, J. C. C., Nepomuceno, M. F., Ávila, M. R. C., Lara, G. B., \& Carvalho-Freitas, M. N. (2008). Análise da produção acadêmica nacional dos últimos 20 anos sobre a inserção das pessoas portadoras de deficiência no mercado de trabalho. In: M. N. Carvalho-Freitas, \& A. L. Marques. Trabalho e pessoas com deficiência: pesquisas, práticas e instrumentos de diagnóstico (pp. 23-41.). Curitiba: Juruá Editora.

Tanaka, E. D. O. (2007). O desenvolvimento de uma escala de atitudes sociais em relação ao trabalho da pessoa com deficiência. Tese de doutorado em Educação, Universidade Estadual Paulista, Marília, SP, Brasil.

Vitor, M. R. C. A. (2011). Valores organizacionais e concepções de deficiência: A percepção das pessoas com deficiência. Dissertação de mestrado, Universidade Federal de São João del-Rei, São João del-Rei, MG, Brasil. 
Recebido em: 19/04/2013

Notas:

* Agradecimentos ao CNPq pelo apoio ao financiamento da pesquisa.

1 Será utilizado o termo pessoa com deficiência, pois tem sido considerada, nacional e internacionalmente, a nomenclatura mais adequada por não representar discriminação contra as pessoas que possuem uma deficiência. $\mathrm{O}$ termo deficiente qualifica e reduz a pessoa a um atributo. O termo portador indica a possibilidade de deixar de portar, o que, na maioria dos casos, não é uma possibilidade para essas pessoas. Também, desde a divulgação da Portaria 2.344/2010, o termo pessoa com deficiência passou a ser utilizado na legislação e em todos os documentos oficiais do governo brasileiro.

2 Não foram solicitados, na presente pesquisa, dados que caracterizassem as empresas, pois o foco da investigação eram as crenças, atitudes e práticas de RH na percepção dos profissionais de Recursos Humanos.

3 A religião foi considerada uma informação importante, pois a concepção espiritual foi utilizada como uma das crenças possíveis de serem compartilhadas entre os participantes. 
